\begin{tabular}{llll}
\hline Submission: 19/Feb/2021; & $1^{\text {st }}$ round notif.: 05/May/2021; & New version: 24/May/2021; & $2^{\text {nd }}$ round notif.: 30/Jun/2021; \\
Camera ready: 12/Jul/2021; & Edition review: 21/Jul/2021; & Available online: 06/Aug/2021; & Published: 06/Aug/2021; \\
\hline
\end{tabular}

\title{
Revisión de Tecnologías Educativas que Fomentan la Lectura de Comprensión Autónoma
}

\author{
Title: Review of Educational Technologies that Promote Reading for Autonomous \\ Comprehension
}

Adelina Escobar-Acevedo

Benemérita Universidad Autónoma de Puebla

Facultad de Ciencias de la Computación

Doctorado en Ingeniería del Lenguaje y del Doctorado en Ingeniería del Lenguaje y del

Conocimiento

adeesa32@gmail.com

\author{
Josefina Guerrero-García \\ Benemérita Universidad Autónoma de Puebla \\ Facultad de Ciencias de la Computación \\ Conocimiento \\ josefina.guerrero@correo.buap.mx
}

\begin{abstract}
Resumen
La lectura es una actividad importante tanto en la vida diaria como en la académica. A pesar de que los planes curriculares marcan objetivos bien definidos para cada grado de estudios, la lectura no se aplica extensivamente en el aula por ser altamente demandante en carga cognitiva y tiempo. Fuera del aula, los estudiantes carecen de la guía del docente, si bien pueden hacer uso de otros recursos y estrategias como hacer anotaciones, elaborar diagramas, redactar resúmenes, entre otras, para comprender los textos. El apoyo a la comprensión lectora es un desafío en el ámbito tecnológico, la tarea se encuentra dentro de los dominios llamados mal definidos, donde no existe una única respuesta correcta. En este trabajo se presenta una revisión parcial con el objetivo de identificar tecnología educativa propuesta en los últimos años que contribuye directa o indirectamente a la comprensión lectora autónoma. Se hace una breve comparación indicando objetivos y características. Finalmente, se señalan los retos futuros.
\end{abstract}

Palabras-Clave: Comprensión lectora; Tecnologías educativas; Tutores Inteligentes.

\begin{abstract}
Reading is an important activity in both daily and academic life. Although the curricular plans set well-defined objectives for each degree of study, reading is not applied extensively in the classroom because it is highly demanding in terms of cognitive load and time. Outside the classroom, students lack the teacher's guidance, although they can use resources and strategies such as making annotations, drawing diagrams, writing summaries, among others, to understand the texts. Support for reading comprehension is a challenge in the technological field, the task is found within the ill-defined domains, where there is no single correct answer. This work presents a partial review that aims to identify educational technology proposed in recent years that contributes directly or indirectly to autonomous reading comprehension. A brief comparison is made indicating objectives and characteristics. Finally, future challenges are outlined.

Keywords: Reading Comprehension; Educational Technologies; Intelligent Tutor Systems.
\end{abstract}

Cite as: Escobar-Acevedo, A., \& Guerrero-García, J. (2021). Revisión de Tecnologías Educativas que Fomentan la Lectura de Comprensión Autónoma. Revista Brasileira de Informática na Educação, 29, 980-992. DOI: 10.5753/RBIE.2021.29.0.980 


\section{Introducción}

La lectura es una actividad esencial en la vida cotidiana. Cada nivel educativo tiene objetivos curriculares con metas claras, desde la decodificación en preescolar hasta la capacidad de realizar argumentación crítica a niveles de posgrado. Típicamente, se establecen actividades de prelectura para activar conocimientos previos, de apoyo durante la lectura que resuelven dudas específicas y post lectura para determinar el grado de comprensión de los lectores.

Un docente puede guiar a un grupo de alumnos con diferentes características haciendo uso de estrategias y su propia experiencia; sin embargo, leer requiere tiempo y carga cognitiva por lo que su aplicación en el aula es reducida. Fuera del aula los alumnos no cuentan con la guía del docente, pero pueden hacer uso de recursos y estrategias; como usar diccionarios, hacer anotaciones, elaborar diagramas, redactar resúmenes, entre otras; para apoyar su comprensión.

La era digital ha modificado nuestra vida diaria permanentemente incluyendo nuestra forma de leer. La creciente conectividad, libros electrónicos, almacenamiento en la nube y otros desarrollos tecnológicos han facilitado la portabilidad de la lectura e incluso cambiado las estrategias de enseñanza en las escuelas. Si bien las habilidades para el Siglo XXI contemplan un apartado explícito de habilidades tecnológicas, de la información y medios (Information, Media \& Technology Skill) (Fadel et al., 2008), con la reciente pandemia han cobrado relevancia al grado de impactar en el éxito académico.

La inhabilitación de actividades presenciales permite observar tres factores relacionados con la tecnología que impactan en la educación. El primero se refiere a las habilidades digitales, en el caso más extremo el analfabetismo digital, que se refiere a "todas aquellas personas que desarrollan sus actividades sin vincularse con tecnologías o medios digitales... factor que influye en el ámbito profesional, personal y social" (Icaza-Álvarez et al., 2019). El segundo se refiere a las capacidades de conectividad del lugar de residencia, por ejemplo, en México el último censo del Instituto Nacional de Estadística y Geografía (INEGI) reveló que sólo el $56.7 \%$ de los hogares cuenta con acceso a Internet; con una desproporción entre zonas rurales y urbanas (INEGI, 2020). El tercer factor recae sobre el grado de desarrollo de la tecnología educativa utilizada, lo que causa aceptación o rechazo por parte de los usuarios.

En este trabajo se presentan los resultados de una investigación de tecnologías educativas recientes orientadas a apoyar la lectura de comprensión sin el apoyo de un docente. El documento se divide en 5 secciones, en la sección 2 se describe el método para realizar la búsqueda de información; en la sección 3 se presenta una reseña que incluye el objetivo de la tecnología reportada, el método seguido y características particulares. Posteriormente, en la sección 4 se muestra una comparación entre las tecnologías, público para el que va dirigida y características. Finalmente, la sección 5 presenta una discusión del trabajo realizado.

\section{Método}

En la literatura es posible encontrar trabajos en los que se aborda el uso de tecnología para el desarrollo de la lectura; en esta sección se reporta el método aplicado en la revisión, sugerido por Okoli y Schabram (2012) y dividido en cuatro etapas: Planeación, selección, extracción y ejecución. En la etapa de planeación se determinó recuperar artículos de revista y conferencias sobre tecnología relacionada con lectura humana, por lo que eligieron cinco bases de datos: IEEE, Springer, ACM, Scopus y Science Direct.

En la etapa de selección, se impusieron los criterios de búsqueda: Rango de enero de 2015 a octubre de 2020 con las palabras clave "Reading comprehension", sin incluir comprensión 
lectora artificial ("Machine Reading comprehension"), limitado al área de Ciencias Computacionales. Después de cumplir con los criterios establecidos, se recuperaron 1698 registros de los cuales 24 eran duplicados. Sólo se conservaron los registros en inglés o español. Entre los trabajos excluidos se encuentran los que estudian diferencias entre lectura en papel y pantalla, uso de dispositivos electrónicos, efectos de la música durante la lectura y estudios de movimientos oculares. Mediante lectura de títulos, palabras claves y resúmenes, se seleccionaron 89 trabajos candidatos relacionados con tecnología para lectura humana.

En la fase de extracción, se consideran trabajos al menos con pruebas preliminares, las propuestas sin resultados fueron descartadas; se buscó identificar: objetivo, público de interés, tipo y características de la tecnología. En la cuarta fase, de ejecución, se realizó una síntesis de los trabajos y se organizó la información. En total, se reportan 27 estudios cuyo propósito es apoyar a los usuarios en el proceso de lectura de comprensión de forma autónoma.

\section{Tecnología en Fomento a la Lectura de Comprensión}

En los dominios bien definidos, como las matemáticas, existe una única respuesta correcta, pero la lectura y escritura son catalogadas como dominios mal definidos y representan un desafío tecnológico (Jacovina \& McNamara, 2016). El acompañamiento humano es deseable para dar seguimiento adecuado al lector; sin embargo, no siempre está disponible, de forma que se ha buscado automatizar ciertas tareas mediante Procesamiento Natural de Lenguaje y Representación de Conocimiento. Actualmente, hay avances importantes en clasificación y simplificación de textos, recuperación de información, generación automática de preguntas, calificación de resúmenes, entre otras.

\subsection{Sistema de Recomendación}

Los sistemas de recomendación son técnicas y herramientas de software que sugieren elementos al usuario, la intención es apoyarlo en el proceso de toma de decisiones (Ricci et al., 2015). Si bien un sistema de recomendación no apoya directamente la comprensión lectora, se incluyó en esta revisión porque es una de las propuestas para motivar al alumno a efectuar las lecturas, dado que la mayoría de los investigadores reportan desinterés de parte de los usuarios.

Shen et al. (2018) crearon un sistema para teléfonos móviles pensado para estudiantes con conocimiento bajo de idioma inglés considerando el vocabulario como una métrica de evaluación. El sistema recomienda a los alumnos textos de su interés y permite hacer pruebas de vocabulario para determinar si han avanzado de nivel. Se utilizaron lecturas de libros de texto que están previamente etiquetadas por niveles. Para considerar la lectura exitosa, el alumno debe obtener más del $60 \%$ en la prueba de vocabulario. No se ha probado de forma extensa, pero cumple con el objetivo de facilitar a los alumnos lecturas y aprendizaje de vocabulario.

\subsection{Computación Humana}

Dos de las actividades post lectura comunes para identificar comprensión son la elaboración de resúmenes y la creación de mapas conceptuales. Si bien existen avances en la evaluación automática de dichos productos, una solución ingeniosa es recurrir a la llamada "human computation" que por definición se refiere a la interconexión entre humanos y máquinas para procesar información como un sistema en el que todos tienen el mismo propósito (Michelucci, 2013). En este caso, asigna tareas a los usuarios de forma que las contribuciones efectuadas por cada uno incrementan la cantidad de recursos y su calidad. Esta solución ha sido explotada en otros procesos complejos como la traducción. 
Brain Trust es un sistema que asigna textos a los usuarios (Olney \& Cade, 2015). Un estudiante virtual hace preguntas abiertas, propone conceptos clave y mapas mentales que el usuario corrige. De esta forma se promueve la materialización de la comprensión lectora mientras se generan materiales para los textos. La ventaja principal es la distribución del tiempo invertido en la creación de recursos; sin embargo, por ser tareas que requieren esfuerzo, los usuarios se cansan rápido de realizarlas repetitivamente. Los autores incrementan la autonomía del usuario como una forma de motivarlo.

$i$-Read es un sistema web que registra las anotaciones que hacen los alumnos en los textos (Omheni \& Kacem, 2016). Además, las anotaciones se categorizan en tres niveles de lector. El objetivo es que los usuarios con bajo nivel de conocimiento puedan acceder a las anotaciones realizadas por los usuarios de nivel superior y que dicha información les sirva de apoyo. Se han hecho pruebas a pequeña escala.

\subsection{Mapas Conceptuales y Argumentativos}

La creación de mapas conceptuales consiste en organizar la información indicando la relación entre conceptos encontrados en el texto (Novak \& Cañas, 2008). Los mapas argumentativos son jerárquicos, en forma piramidal y se usan para desarrollar pensamiento crítico. Para poder realizar los mapas, la propuesta tecnológica debe integrar una herramienta gráfica o en su defecto utilizar la estructura ya definida permitiendo que el usuario arrastre objetos a la posición deseada. Durante la búsqueda se encontraron al menos dos trabajos que hacen uso exclusivo de esta actividad.

Morfidi y Mikropoulos (2017) realizaron un estudio con niños de quinto grado de primaria en Grecia creando mapas conceptuales para mejorar su comprensión. La necesidad surgió dentro de un proyecto de desastres naturales conformado por cuatro lecciones con textos de 200 a 300 palabras. Al finalizar la lectura, los niños arrastran los conceptos a la posición deseada dentro de un mapa previamente creado por expertos en el área, siguiendo los principios de Novak y Cañas (2008). Los resultados indican más aciertos en el cuestionario en el grupo que tuvo la herramienta contra el grupo con lectura tradicional.

CAERS (Computer aided argumentative essay reading system), creado para niños de sexto grado de primaria, tiene el objetivo de apoyar a los estudiantes en la comprensión de ensayos argumentativos (Chiang et al., 2016). El sistema tiene dos funciones principales, la primera es que los estudiantes conozcan la organización de un ensayo argumentativo. La segunda es crear un mapa conceptual o argumentativo para entender la relación entre los elementos (hechos, razones y evidencia). Los alumnos pueden elegir el texto que desean trabajar, al terminar la lectura crean su mapa, finalmente los alumnos pueden visualizar un mapa modelo creado por los docentes y la explicación de por qué se elaboró así. Los docentes pueden subir material que deseen que los alumnos lean y monitorear su práctica individual. Para las pruebas se dispuso de seis clases para entrenamiento, textos de 450 a 550 palabras con 20 preguntas de opción múltiple. Los resultados indican que, para la tarea, los mapas argumentativos fueron más claros para los estudiantes. Remarca que la ventaja de usar mapas es que los alumnos pueden usar gráficos que refuerzan su comprensión.

\subsection{Máquina de Conocimiento}

La Máquina de Conocimiento (KM: The Knowledge Machine), acorde a sus autores, combina un lenguaje de representación de conocimiento con una representación orientada a objetos con reglas deductivas, además, se puede exportar a una ontología. Más información sobre este desarrollo se puede consultar directamente en la página "https://www.cs.utexas.edu/users/mfkb/km.html". 
Inquire Biology es un libro de texto inteligente creado para estudiantes universitarios (Chaudhri et al., 2015). Está fundamentado en una Máquina de Conocimiento llamada AURA en la que participaron biólogos y docentes de biología para incluir 6000 conceptos y más de 100,000 reglas. Permite al alumno navegar por el libro, subrayar oraciones y hacer preguntas directamente. El sistema puede generar preguntas sobre los textos subrayados, algunas directas y otras que requieren conocimiento de una o más secciones del libro. Cada palabra del libro de texto está ligada a un concepto y puede mostrar un resumen relacionado. Este trabajo es uno de los productos de la base de conocimiento creada. Las pruebas indicaron que los estudiantes que trabajaron con el libro obtenían más aciertos en las evaluaciones que aquellos que leían texto plano.

\subsection{Tutores Inteligentes}

Los tutores inteligentes son sistemas complejos cuyo objetivo es apoyar al estudiante durante su aprendizaje. Entre las tareas que un tutor podría incluir se encuentran: proveer materiales a los estudiantes de acuerdo con su estado de aprendizaje, dar seguimiento a sus avances, retroalimentarlos, proporcionar material de apoyo (Cataldi \& Lage, 2009). Cada una de estas tareas implica procesamiento específico de datos, por ejemplo, para conocer el estado de aprendizaje se requieren datos del desempeño, tradicionalmente un umbral determina si el ejercicio se considera aprobado o no. Para la retroalimentación, el sistema debe ser capaz de identificar errores o asignar algún criterio de calidad al trabajo del usuario y así enviar el mensaje o material de apoyo adecuados. En la presente revisión se encontraron cuatro tutores inteligentes para la lectura de comprensión: AutoTutor, iSTART, ITSS y EMBRACE. Cada tutor está dirigido a público objetivo específico y utiliza una estrategia pedagógica definida.

\subsubsection{AutoTutor}

Es el tutor más antiguo identificado en esta revisión que sigue reportando avances, lanzado en 1997, su objetivo es equiparase con un tutor humano y ha sido origen de otros tutores en distintos dominios. Puede consultarse y probarse en la página “http://ace.autotutor.org/IISAutotutor/index.html". La versión estudiada aquí está dirigida a adultos con bajo nivel de lectoescritura y se aplica en un centro de aprendizaje llamado CSAL (Center for the Study of Adult Literacy). Entre las características relevantes del público objetivo se encuentran: niveles de estudios heterogéneos, habilidades informáticas reducidas o carentes, variedad demográfica, algunos no dominan el idioma inglés de forma nativa (Shi et al., 2018).

AutoTutor tiene dos agentes conversacionales con voz automatizada. El agente femenino toma el rol de docente y el masculino funge como compañero de clase, de forma que el usuario se mantenga motivado y acompañado durante la interacción. El agente docente hace preguntas tanto al alumno humano como al alumno digital, los diálogos dependen de la respuesta del alumno, este enfoque ha resultado suficiente para que los alumnos intenten hacer preguntas directamente al agente profesor; lo que aún no es posible (Fang et al., 2019).

Las instrucciones son auditivas e incluye práctica de habilidades informáticas básicas como dar clic y usar la barra de desplazamiento. Se cuidó una interfaz intuitiva, sencilla, con uso reducido de teclado y basada en principios de Interacción humano-computadora; es accesible desde cualquier conexión a internet, lo que facilita su aceptación. AutoTutor cuenta con 35 lecciones interactivas, cada una inicia con un video, toma entre 10 y 50 minutos e incluye de 10 a 35 preguntas de opción múltiple. El alumno tiene libertad de elegir las lecturas e incluso puede jugar Jeopardy con los agentes (Baer et al., 2016). Actualmente se enfocan en lograr un currículo adaptativo de forma que el usuario no tenga que ver todas las lecciones, (Fang et al., 2019). 
Un estudio buscó la preferencia de retroalimentación y su impacto en el desempeño del alumno. La retroalimentación epistémica informa si la respuesta fue correcta o no. La emocional incluye el nombre del alumno y un mensaje de felicitación o tristeza. La neutra manda un OK en cada respuesta sin indicar si es correcta. Se identificó, con cámaras, que la epistémica desencadena emociones más extremas mientras que una encuesta arrojó que los usuarios prefieren la emocional, ambas son superiores a la retroalimentación neutra (Feng et al., 2015). En otro estudio, con alumnos cuyo inglés es el segundo idioma, se modificaron a los agentes para mantener diálogos con lenguaje formal, informal y mixto, en el mixto el agente profesor usa lenguaje formal y el agente estudiante informal. No se identificó cambio en el aprendizaje, pero si en el compromiso de los alumnos cuando se usó lenguaje formal (Li \& Graesser, 2017).

\subsection{2 iSTART}

iSTART (Interactive Strategy Training for Active Reading and Thinking) es un sistema tutor inteligente lanzado en 2004 basado en SERT (Self Explanation Reading Training), estrategia metacognitiva en la cual los usuarios escriben una breve auto explicación posterior a la lectura de un texto complejo. Las producciones son comparadas con los textos originales para asignar un puntaje entre 0 y 3 usando Latent Semantic Analysis (LSA), Posee lecciones en video mostrando las ventajas y la forma de realizar las auto explicaciones. Existe una sección de práctica guiada con textos cortos y ejemplos antes de la práctica libre (McCarthy et al., 2020). El repositorio de iSTART incluye 100 textos de áreas principalmente de ciencias, medicina e historia y está dirigido a estudiantes de preparatoria y primer grado universitario.

Es el tutor que establece criterios más rigurosos en los experimentos para comprobar las variables que inciden en la lectura de comprensión. Se aplican encuestas que pueden ser demográficas, de lectura de comprensión, de aversión a la lectura, de conocimientos generales entre otras. Los resultados se comparan con un grupo control y el número de sesiones varía, de una a once, acorde al objetivo.

Una investigación comprobó la relación entre buenos puntajes de la auto explicación y los puntajes de compresión lectora (Allen et al., 2015). Se implementó una forma para diferenciar visualmente un texto de calidad y uno con bajo puntaje (Allen et al., 2017). También se buscaron criterios para que los puntajes asignados automáticamente a las auto explicaciones sean equiparables con los que asignaría un humano (Crossley et al., 2015). Se pidió a los alumnos que escribieran una explicación para sí mismos y una para un compañero, identificando que existen diferencias entre ambas tareas ya que la auto explicación activa procesos cognitivos con textos más cohesivos (Allen et al., 2019). Se intentó hacer consciente al alumno de su umbral de desempeño los resultados no indicaron mejora en la comprensión, pero si en la calidad del escrito (McCarthy et al., 2017). Sobre esto, los autores dicen que la auto explicación es ya una actividad metacognitiva por lo que no requiere sobrecargarse (McCarthy, Likens, et al., 2018). Resultados similares se obtuvieron con la versión iSTART-2 (Snow, McNamara, et al., 2015).

Existen varias versiones, iSTART-ME (ME: Motivationally-Enhanced) da monedas virtuales conforme al desempeño con las que los usuarios pueden personalizar su entorno (Snow, Allen, et al., 2015). Los autores refieren a estudios previos donde incluir distractores afecta el desempeño del estudiante, sin embargo, argumentan que dichos estudios se llevaron a cabo en pocas sesiones y no se puede asegurar que a largo plazo sean contraproducentes. En este caso, los estudiantes tendieron a valuar sus interacciones con los juegos como experiencias positivas, a la vez, este tipo de experimentos permite identificar las tendencias del estudiante sobre su administración de tiempo. Por otra parte, iSTART-2 es una versión gamificada, si bien se obtienen puntajes mayores en el post test, no son diferencias significativas contra la versión sencilla. Los juegos en el estudio realizado no tienen otro efecto que mantener el interés del 
alumno, sin embargo, si demuestra que ambas versiones de iSTART contribuyen a la comprensión lectora (Jacovina et al., 2016).

En iSTART-ALL (Johnson et al., 2017) el público objetivo son adultos con bajo nivel literario, similar a AutoTutor. Se agregaron lecciones sobre habilidades tecnológicas y 60 textos relevantes en la vida diaria (tecnología, salud, familia) con niveles entre 3er y 8vo grados acorde a nivel escolar. En total las lecciones alcanzan 26 minutos de video en total integrando voz y presentaciones en Prezi. El tutor está basado en las estrategias de lectura para adultos reportadas por Hock y Mellard (2005). La gamificación incluye un teléfono virtual personalizable. 66\% de aciertos indica que los niveles de lectura son adecuados. iSTART-E (McCarthy, Soto, et al., 2018) es una versión traducida al español que se aplicó en Argentina y Chile.

La última versión, iSTART-3 (McCarthy et al., 2020) fue creada para proporcionar a los estudiantes textos de forma adaptativa conforme a su nivel de lectura, incluye nueve niveles de dificultad equivalentes al año escolar (de 6to a 14vo). Alumnos con menores niveles de lectura se beneficiaron más con la adaptación automática. Los alumnos con alto nivel reportaron una ligera desventaja. El Módulo StairStepper ya se había desarrollado previamente para tener la función de una 'escalera' permitiendo a los estudiantes subir escalones o bajarlos conforme a su desempeño (Perret et al., 2017).

\subsubsection{ITSS}

ITSS (Wijekumar et al., 2017) Intelligent Tutoring System for the Structure Strategy, es un tutor inteligente creado para niños de cuarto y quinto grado de primaria. Es precisamente en el cuarto grado cuando los niños inician la lectura de contenido por lo que enfrentan nuevos retos de comprensión lectora. La intención es instruir a los niños sobre cómo leer, seleccionar las ideas principales, codificar estructuras de memoria estratégicas, hacer inferencias y monitorear la comprensión durante la lectura.

El modelo pedagógico en el que está basado se ha desarrollado por más de 35 años de investigación (Meyer et al., 1980) y consiste en utilizar la estructura del texto para organizar la información. Utiliza 5 estructuras de texto: Comparación, problema y solución, causa y efecto, secuencia y descripción. Todos los textos fueron seleccionados basándose en grados, temas y tipos de tarea. Existe un agente pedagógico animado que interactúa con los niños como docente, explicando, haciendo preguntas y guiando al alumno. El tutor ha sido probado con miles de alumnos y demuestra diferencia significativa en las pruebas estandarizadas al aplicarlo.

\subsubsection{EMBRACE}

EMBRACE (Walker et al., 2017) es un libro interactivo que cuenta con un tutor inteligente. Fue creado para niños de primaria cuyo inglés es su segundo idioma. Al inicio de cada capítulo muestra una lista de palabras recuperadas de la lección anterior, de la lección por estudiar y aquellas en las que el estudiante demostró habilidad inferior a 0.80 en actividades anteriores; no se muestran más de ocho palabras para evitar saturar al alumno. Los niños deben seleccionar y mover objetos acordes a la historia. Cuando no se cumple con las tareas requeridas, las acciones se deshacen. La interacción permite identificar la comprensión del texto y determinar si el problema es de vocabulario o de sintaxis. El diseño del tutor está basado en los principios de Anderson et al., (1995) que incluyen desglosar la tarea en reglas de manipulación, proveer retroalimentación inmediata, adaptar la complejidad, entre otras. 


\section{Resultados}

Se identificaron diez tecnologías que apoyan al estudiante en la lectura de comprensión. Se pueden agrupar por el tipo de apoyo en indirectos: Sistemas de Recomendación y Computación Humana; y directos: Mapas Conceptuales y Argumentativos, Máquina de Conocimiento y Tutores Inteligentes. Para presentar una síntesis, la Tabla 1 reporta las tecnologías identificadas con el público al que va dirigida, sus objetivos y características.

El sistema móvil de recomendación se clasificó como apoyo indirecto porque, si bien no interviene directamente en el proceso de lectura, permite al usuario elegir el material con el que trabaja considerando el vocabulario. Esto puede tener efectos positivos en la motivación, ya que el desinterés de los usuarios se reporta repetitivamente en las investigaciones sobre lectura.

Los trabajos de Computación humana caen en un apoyo indirecto-colaborativo. Mediante la interacción humana se recopila información, de forma que, un gran grupo de estudiantes al cumplir con la tarea encargada, por ejemplo, realizar un mapa mental, va perfeccionando gradualmente el producto. Posteriormente, los trabajos realizados por compañeros predecesores pueden utilizarse de dos formas directas: la primera como ejemplo a estudiantes que no alcanzan los estándares y la segunda como marco de referencia que permita evaluar las tareas de futuros usuarios.

En los apoyos directos se colocaron primero las tecnologías que facilitan la creación de mapas conceptuales y argumentativos dado que estas actividades permiten al lector reflexionar sobre lo leído, organizar sus ideas y expresarlas, contribuyendo con esto a la comprensión. Por otra parte, la máquina de conocimiento juega un papel importante para el proceso de apoyo durante la lectura, tal cual lo haría un docente en el aula respondiendo preguntas de los alumnos para definir vocabulario o profundizar en conceptos.

Los tutores inteligentes, dependiendo del objetivo del tutor pueden apoyar en cualquiera o todas las fases de lectura. Esta revisión identificó cuatro tutores, dos de ellos con amplio desarrollo; AutoTutor hace uso de agentes conversacionales, iSTART califica automáticamente los escritos de los alumnos, ITSS facilita la organización de ideas y EMBRACE desglosa las instrucciones de forma que puede identificar tipo de error, presentar textos simplificados y dar seguimiento. Los propósitos de los tutores son diferentes en cuanto al público objetivo y las habilidades a desarrollar.

\section{Discusión}

En este trabajo se identificaron tecnologías que apoyan directa o indirectamente a la lectura de comprensión, con especial interés en los estudiantes con bajo desempeño. Considerando que el lenguaje es complejo tanto para los humanos como para las computadoras, los desarrollos actuales hacen uso de estrategias creativas, como la computación humana, para apoyar a los alumnos reduciendo el esfuerzo humano de creación de materiales.

Como la lectura requiere concentración, tiempo y esfuerzo, las investigaciones suelen mencionar el compromiso y la motivación en las discusiones. Recomendar textos, permitir libre navegación, incluir actividades lúdicas e integrar agentes conversacionales se han probado para mantener la atención de los usuarios. Futuros desarrollos deben adaptarse más a los intereses del usuario, logrando experiencias positivas conforme se busca su aprendizaje. 
Tabla 1: Tecnologías identificadas de apoyo a la lectura.

\begin{tabular}{|c|c|c|c|c|c|}
\hline Tipo & Nombre & $\begin{array}{l}\text { Público } \\
\text { objetivo }\end{array}$ & Tecnología & Objetivo & Características \\
\hline \multirow{3}{*}{ 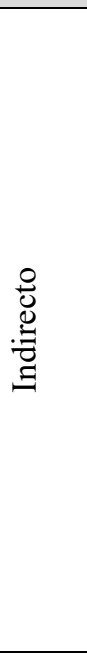 } & $<$ no indica $>$ & General & $\begin{array}{l}\text { Sistema de } \\
\text { recomendación }\end{array}$ & $\begin{array}{l}\text { Motivar la lectura } \\
\text { e incrementar } \\
\text { vocabulario. }\end{array}$ & $\begin{array}{l}\text {-Provee material de interés del } \\
\text { lector considerando vocabulario. }\end{array}$ \\
\hline & Brain Trust & General & $\begin{array}{l}\text { Computación } \\
\text { Humana }\end{array}$ & $\begin{array}{l}\text { Promover la } \\
\text { comprensión } \\
\text { mientras se } \\
\text { generan y } \\
\text { perfeccionan } \\
\text { materiales. }\end{array}$ & $\begin{array}{l}\text {-Distribuye entre los usuarios } \\
\text { tareas como creación de mapas } \\
\text { conceptuales o resúmenes. }\end{array}$ \\
\hline & i-Read & General & $\begin{array}{l}\text { Computación } \\
\text { Humana }\end{array}$ & $\begin{array}{l}\text { Apoyar a los } \\
\text { lectores } \\
\text { deficientes. }\end{array}$ & $\begin{array}{l}\text {-Registra las anotaciones de los } \\
\text { lectores en el texto. } \\
\text { - Clasifica al lector en tres } \\
\text { niveles. } \\
\text { - Las anotaciones de lectores } \\
\text { altos podrían apoyar a los } \\
\text { deficientes. }\end{array}$ \\
\hline \multirow{7}{*}{ 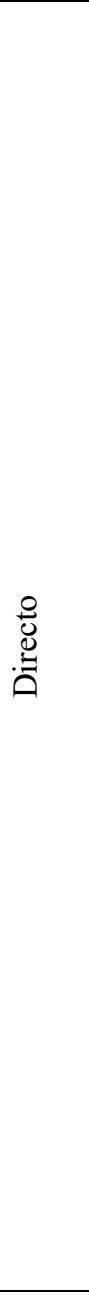 } & $<$ no indica $>$ & $\begin{array}{l}\text { Niños de 5to } \\
\text { grado }\end{array}$ & $\begin{array}{l}\text { Aplicación } \\
\text { para mapas } \\
\text { conceptuales }\end{array}$ & $\begin{array}{l}\text { Organizar } \\
\text { información de } \\
\text { forma gráfica. }\end{array}$ & $\begin{array}{l}\text {-Permite completar mapas ya } \\
\text { creados mediante arrastrar y } \\
\text { soltar. }\end{array}$ \\
\hline & CAERS & $\begin{array}{l}\text { Niños de 6to } \\
\text { grado }\end{array}$ & $\begin{array}{l}\text { Aplicación } \\
\text { para mapas } \\
\text { conceptuales y } \\
\text { argumentativos }\end{array}$ & $\begin{array}{l}\text { Mejorar la } \\
\text { comprensión de } \\
\text { ensayos } \\
\text { argumentativos. }\end{array}$ & $\begin{array}{l}\text {-Permite la creación de mapas } \\
\text { conceptuales y argumentativos. } \\
\text {-Al finalizar se visualizan mapas } \\
\text { modelo y explica cómo se } \\
\text { realizaron. }\end{array}$ \\
\hline & $\begin{array}{l}\text { Inquire } \\
\text { Biology }\end{array}$ & $\begin{array}{l}\text { Estudiantes } \\
\text { universitarios }\end{array}$ & $\begin{array}{l}\text { Máquina de } \\
\text { conocimiento }\end{array}$ & $\begin{array}{l}\text { Profundizar } \\
\text { conceptos. }\end{array}$ & $\begin{array}{l}\text {-Basado en una Máquina de } \\
\text { Conocimiento. } \\
\text {-Contesta y hace preguntas. }\end{array}$ \\
\hline & AutoTutor & Adultos & $\begin{array}{l}\text { Tutor } \\
\text { Inteligente }\end{array}$ & $\begin{array}{l}\text { Igualar a un tutor } \\
\text { humano. }\end{array}$ & $\begin{array}{l}\text { - Lecciones secuenciales. } \\
\text { - Agentes conversacionales de } \\
\text { soporte. }\end{array}$ \\
\hline & iSTART & $\begin{array}{l}\text { Estudiantes de } \\
\text { preparatoria y } \\
\text { universitarios }\end{array}$ & $\begin{array}{l}\text { Tutor } \\
\text { Inteligente }\end{array}$ & $\begin{array}{l}\text { Aplicar auto } \\
\text { explicación como } \\
\text { estrategia de } \\
\text { comprensión } \\
\text { lectora. }\end{array}$ & $\begin{array}{l}\text { - Lecciones con estrategias } \\
\text { lectoras. } \\
\text { - Estrategia de auto explicación. } \\
\text { - Calificación automática de auto } \\
\text { explicaciones. }\end{array}$ \\
\hline & ITSS & $\begin{array}{l}\text { Niños } 4 \text { y } 5 \text { to } \\
\text { grado }\end{array}$ & $\begin{array}{l}\text { Tutor } \\
\text { Inteligente }\end{array}$ & $\begin{array}{l}\text { Enseñar a } \\
\text { organizar ideas. }\end{array}$ & $\begin{array}{l}\text {-Lecciones sobre cómo } \\
\text { identificar ideas principales. } \\
\text {-Permite la práctica. }\end{array}$ \\
\hline & EMBRACE & $\begin{array}{l}\text { Niños segundo } \\
\text { idioma }\end{array}$ & $\begin{array}{l}\text { Tutor } \\
\text { Inteligente }\end{array}$ & $\begin{array}{l}\text { Guiar a los niños } \\
\text { en la comprensión } \\
\text { lectora. }\end{array}$ & $\begin{array}{l}\text {-Lecciones sobre estrategias } \\
\text { lectoras. } \\
\text {-Retroalimentación inmediata } \\
\text { durante la interacción con el } \\
\text { sistema. } \\
\text {-Seguimiento del vocabulario y } \\
\text { sintaxis. } \\
\text {-Si el desempeño del alumno } \\
\text { decae, muestra versiones } \\
\text { simplificadas del texto. }\end{array}$ \\
\hline
\end{tabular}

Algunas de las tecnologías reportan incremento en los puntajes de comprensión lectora de los usuarios incluso en versiones sencillas, lo que significa que su uso es efectivo y son una opción de lectura fuera del aula. Lamentablemente, el costo en tiempo y esfuerzo para su desarrollo es todavía un factor negativo. Además, se deben considerar la funcionalidad, la conectividad y las habilidades tecnológicas de alumnos y docentes. 
Una observación importante es que los trabajos aquí reportados se desarrollaron principalmente para el idioma inglés. Lo que representa un área de oportunidad para los investigadores debido a que la experiencia con iSTART-E ya identificó que trasladar recursos de un idioma a otro, no es trivial.

Como conclusión, la tecnología educativa permite mejorar la enseñanza y facilitar el acceso a la educación. La educación actual tiende a que las instituciones y los profesores estén cada vez más conectados, haciendo uso de esas tecnologías para transmitir el conocimiento y mejorar el aprendizaje de sus alumnos.

\section{Agradecimientos}

Este trabajo fue realizado bajo apoyo de CONACYT con la beca 235249.

\section{Referencias}

Allen, L. K., Likens, A., Perret, C., \& McNamara, D. S. (2017). What'd you say again? Recurrence quantification analysis as a method for analyzing the dynamics of discourse in a reading strategy tutor. Proceedings of the Seventh International Learning Analytics \& Knowledge Conference, 373-382. doi: 10.1145/3027385.3027445 [GS search]

Allen, L. K., Perret, C., Mills, C., \& McNamara, D. S. (2019). Are you talking to me? Multidimensional language analysis of explanations during reading. Proceedings of the 9th International Conference on Learning Analytics \& Knowledge (LAK19), 116-120. doi: $\underline{10.1145 / 3303772.3303835}$ [GS search]

Allen, L. K., Snow, E. L., \& McNamara, D. S. (2015). Are you reading my mind? Modeling students' reading comprehension skills with natural language processing techniques. $A C M$ International Conference Proceeding Series, 16-20-Marc, 246-254. doi: $\underline{10.1145 / 2723576.2723617}$ [GS search]

Anderson, J. R., Corbett, A. T., Koedinger, K. R., \& Pelletier, R. (1995). Cognitive Tutors: Lessons Learned. Journal of the Learning Sciences, 4(2), 167-207. doi:

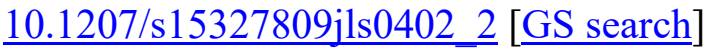

Baer, W. O., Cheng, Q., McGlown, C., Gong, Y., Cai, Z., \& Graesser, A. C. (2016). Using Virtual Agents to Deliver Lessons in Reading Comprehension to Struggling Adult Learners (D. Traum, W. Swartout, P. Khooshabeh, S. Kopp, S. Scherer, \& A. Leuski (Eds.); Vol. 10011, pp. 516-518). Springer International Publishing. doi: 10.1007/978-3-319-47665$\underline{0} 67$ [GS search]

Cataldi, Z., \& Lage, F. J. (2009). Sistemas tutores inteligentes orientados a la enseñanza para la comprensión. Revista Electrónica de Tecnología Educativa, 1-19. [GS search]

Chaudhri, V. K., Overholtzer, A., \& Spaulding, A. (2015). An Intelligent Textbook that Answers Questions (P. Lambrix, E. Hyvönen, E. Blomqvist, V. Presutti, G. Qi, U. Sattler, Y. Ding, \& C. Ghidini (Eds.); Vol. 8982, Issue 2, (pp. 131-135). Springer International Publishing. doi:10.1007/978-3-319-17966-7 16 [GS search]

Chiang, K., Fan, C., Liu, H., \& Chen, G. (2016). Effects of a computer-assisted argument map learning strategy on sixth-grade students' argumentative essay reading comprehension. Multimedia Tools and Applications, 75(16), 9973-9990. doi: 10.1007/s11042-015-2904-y [GS search]

Crossley, S., Allen, L. K., Snow, E. L., \& McNamara, D. S. (2015). Pssst... textual features... 
there is more to automatic essay scoring than just you! Proceedings of the Fifth International Conference on Learning Analytics And Knowledge - LAK '15, 203-207. doi: $\underline{10.1145 / 2723576.2723595}$ [GS search]

Fadel, C., Lead, G., \& Systems, C. (2008). 21st Century Skills: How can you prepare students for the new Global Economy? May. [GS search]

Fang, Y., Lippert, A., Cai, Z., Hu, X., \& Graesser, A. C. (2019). A Conversation-Based Intelligent Tutoring System Benefits Adult Readers with Low Literacy Skills. In Lecture Notes in Computer Science (including subseries Lecture Notes in Artificial Intelligence and Lecture Notes in Bioinformatics): Vol. 11597 LNCS (pp. 604-614). Springer International Publishing. doi: 10.1007/978-3-030-22341-0 47 [GS search]

Feng, S., Stewart, J., Clewley, D., \& Graesser, A. C. (2015). Emotional, Epistemic, and Neutral Feedback in AutoTutor Trialogues to Improve Reading Comprehension. In Lecture Notes in Computer Science (including subseries Lecture Notes in Artificial Intelligence and Lecture Notes in Bioinformatics) (Vol. 9112, pp. 570-573). doi: 10.1007/978-3-319-19773-9 64 [GS search]

Hock, M., \& Mellard, D. (2005). Reading Comprehension Strategies for Adult Literacy Outcomes. Journal of Adolescent \& Adult Literacy, 49(3), 192-200. doi: 10.1598/JAAL.49.3.3 [GS search]

Icaza-Álvarez, D. O., Campoverde-Jiménez, G. E., Verdugo-Ormaza, D. E., \& Arias-Reyes, P. D. (2019). El analfabetismo tecnológico o digital. Polo Del Conocimiento, 4(2), 393. doi 10.23857/pc.v4i2.922 [GS search]

INEGI. (2020). Estadística a propósito del día mundial del internet (17 de mayo) datos nacionales. Comunicado de Prensa Núm. 216/20, 2019, 1-8. Descargado de https://www.inegi.org.mx/contenidos/saladeprensa/aproposito/2020/eap_internet20.pdf

Jacovina, M. E., \& McNamara, D. S. (2016). Intelligent tutoring systems for literacy: Existing technologies and continuing challenges. In Intelligent Tutoring Systems: Structure, Applications and Challenges (Issue January 2016, pp. 153-174). [GS search]

Jacovina, M. E., Tanner Jackson, G., Snow, E. L., \& McNamara, D. S. (2016). Timing GameBased Practice in a Reading Comprehension Strategy Tutor. In A. Micarelli, J. Stamper, \& K. Panourgia (Eds.), Intelligent Tutoring Systems (Vol. 9684, pp. 59-68). Springer International Publishing. doi: 10.1007/978-3-319-39583-8_6 [GS search]

Johnson, A. M., Guerrero, T. A., Tighe, E. L., \& McNamara, D. S. (2017). iSTART-ALL: Confronting Adult Low Literacy with Intelligent Tutoring for Reading Comprehension. In Aied 2017 (Vol. 1, Issue August 2018, pp. 125-136). doi: 10.1007/978-3-319-61425-0_11 [GS search]

Li, H., \& Graesser, A. (2017). Impact of Pedagogical Agents' Conversational Formality on Learning and Engagement. 1, 188-200. doi: 10.1007/978-3-319-61425-0_16 [GS search]

McCarthy, K. S., Jacovina, M. E., Snow, E. L., Guerrero, T. A., \& McNamara, D. S. (2017). iSTART Therefore I Understand: But Metacognitive Supports Did not Enhance Comprehension Gains. International Conference on Artificial Intelligence in Education, 1, 201-211. doi: 10.1007/978-3-319-61425-0_17 [GS search]

McCarthy, K. S., Likens, A. D., Johnson, A. M., Guerrero, T. A., \& McNamara, D. S. (2018). Metacognitive Overload!: Positive and Negative Effects of Metacognitive Prompts in an Intelligent Tutoring System. International Journal of Artificial Intelligence in Education, 28(3), 420-438. doi: 10.1007/s40593-018-0164-5 [GS search] 
McCarthy, K. S., Soto, C., Malbrán, C., Fonseca, L., Simian, M., \& McNamara, D. S. (2018). iSTART-E: Reading Comprehension Strategy Training for Spanish Speakers. In International Conference on Artificial Intelligence in Education, 1(August), 215-219. doi: 10.1007/978-3-319-93846-2 39 [GS search]

McCarthy, K. S., Watanabe, M., Dai, J., \& McNamara, D. S. (2020). Personalized learning in iSTART: Past modifications and future design. Journal of Research on Technology in Education, 52(3), 301-321. doi: 10.1080/15391523.2020.1716201 [GS search]

Meyer, B. J. ., Brandt, D. M., \& Bluth, G. J. (1980). Use of Top-Level Structure in Text: Key for Reading Comprehension of Ninth-Grade Students. Reading Research Quarterly, 16(1), 72-103. [GS search]

Michelucci (Ed.). (2013). Handbook of Human Computation. Springer New York. doi: 10.1007/978-1-4614-8806-4 [GS search]

Morfidi, E., Mikropoulos, A., \& Rogdaki, A. (2018). Using concept mapping to improve poor readers' understanding of expository text. Education and Information Technologies, 23(1), 271-286. doi: 10.1007/s10639-017-9600-7 [GS search]

Novak, J. D., \& Cañas, A. J. (2008). The Theory Underlying Concept Maps and How to Construct and Use Them. [GS search]

Okoli, C., \& Schabram, K. (2012). A Guide to Conducting a Systematic Literature Review of Information Systems Research. SSRN Electronic Journal, 10(2010). doi: 10.2139/ssrn.1954824 [GS search]

Olney, A. M., \& Cade, W. L. (2015). Authoring Intelligent Tutoring Systems Using Human Computation: Designing for Intrinsic Motivation. In D. D. Schmorrow \& C. M. and Fidopiastis (Eds.), Foundations of Augmented Cognition (pp. 628-639). Springer International Publishing. doi: 10.1007/978-3-319-20816-9 60 [GS search]

Omheni, N., \& Kacem, A. H. (2016). "i-Read": A Collaborative Learning Environment to Support Students with Low Reading Abilities. International Conference on Intelligent Tutoring Systems, 9684, 221-226. doi: 10.1007/978-3-319-39583-8 21 [GS search]

Perret, C. A., Johnson, A. M., McCarthy, K. S., Guerrero, T. A., Dai, J., \& McNamara, D. S. (2017). StairStepper: An Adaptive Remedial iSTART Module (pp. 557-560). doi: 10.1007/978-3-319-61425-0 63 [GS search]

Ricci, F., Rokach, L., \& Shapira, B. (Eds.). (2015). Recommender Systems Handbook. Springer US. doi: $10.1007 / 978-1-4899-7637-6$ [GS search]

Shen, W., Lin, J.-M., \& Hong, Z.-W. (2018). An Extensive Reading System Built on the Basis of Comprehensible Input Principles - A Key to Rescuing the Lower-Level EFL University Students' Vocabulary Ability. International Conference on Innovative Technologies and Learning, i, 536-545. doi: 10.1007/978-3-319-99737-7_57 [GS search]

Shi, G., Lippert, A. M., Shubeck, K., Fang, Y., Chen, S., Pavlik, P., Greenberg, D., \& Graesser, A. C. (2018). Exploring an intelligent tutoring system as a conversation-based assessment tool for reading comprehension. Behaviormetrika, 45(2), 615-633. doi: $\underline{10.1007 / \mathrm{s} 41237-}$ $\underline{018-0065-9}$ [GS search]

Snow, E. L., Allen, L. K., Jackson, G. T., \& McNamara, D. S. (2015). Spendency: Students' Propensity to Use System Currency. International Journal of Artificial Intelligence in Education, 25(3), 407-427. doi: 10.1007/s40593-015-0044-1 [GS search]

Snow, E. L., McNamara, D. S., Jacovina, M. E., Allen, L. K., Johnson, A. M., Perret, C. A., Dai, J., Tanner Jackson, G., Likens, A. D., Russell, D. G., \& Weston, J. L. (2015). Promoting 
Metacognitive Awareness within a Game-Based Intelligent Tutoring System. In Lecture Notes in Computer Science (including subseries Lecture Notes in Artificial Intelligence and Lecture Notes in Bioinformatics) (Vol. 9112, pp. 786-789). doi: 10.1007/978-3-319-197739_116 [GS search]

Walker, E., Wong, A., Fialko, S., Restrepo, M. A., \& Glenberg, A. M. (2017). EMBRACE: Applying Cognitive Tutor Principles to Reading Comprehension. In International Conference on Artificial Intelligence in Education 2017 (Vol. 1, pp. 578-581). doi: 10.1007/978-3-319-61425-0_68 [GS search]

Wijekumar, K. K., Meyer, B. J. F., Lei, P., Cheng, W., Ji, X., \& Joshi, R. M. (2017). Evidence of an Intelligent Tutoring System as a Mindtool to Promote Strategic Memory of Expository Texts and Comprehension With Children in Grades 4 and 5. Journal of Educational Computing Research, 55(7), 1022-1048. doi: 10.1177/0735633117696909 [GS search] 